\section{Check for updates}

Cite this: Soft Matter, 2018, 14,653

Received 20th November 2017, Accepted 15th December 2017

DOI: $10.1039 / \mathrm{c} 7 \mathrm{sm} 02286 \mathrm{~g}$

rsc.li/soft-matter-journal

\title{
Nematic line defects in microfluidic channels: wedge, twist and zigzag disclinations $\uparrow$
}

\author{
Hakam Agha and Christian Bahr (D)*
}

\begin{abstract}
We report an experimental study of structural transformations of disclination lines in nematic liquid crystals in microfluidic channels. The anchoring conditions of the channel walls enforce the generation of a disclination line of the wedge type in the absence of flow. The wedge disclination is transformed to a twist disclination by the flow of the nematic liquid crystal in the channel. The application of an electric field perpendicular to the channel axis induces a second transformation to a zigzag shape. The threshold field strength for the second transformation increases with increasing flow velocity. The experimental results are compared to predictions based on model director fields of the different disclination structures.
\end{abstract}

\section{Introduction}

Topological defects play an important role in soft condensed matter physics. ${ }^{1}$ Nematic liquid crystals (NLCs), small rod-shaped organic molecules exhibiting a liquid phase with long range orientational order, show a wealth of different topological defect structures, thereby providing a well suited experimental model system for studies which are significant also to various areas beyond the field of NLCs. Examples range from knot theory ${ }^{2,3}$ over colloid physics ${ }^{4,5}$ to cosmology. ${ }^{6}$

Topological defects may be classified as points, lines or walls. The present study is concerned with line defects in NLCs. Whereas line defects in solids are in most cases dislocations (i.e., the defect is characterized by a linear displacement in the structure of the material), line defects in NLCs are disclinations, which are characterized by a rotational displacement of the structure. ${ }^{7-9}$ The orientational order in NLCs is described by a unit vector, the director $\vec{n}$, which describes the direction along which the rod shaped molecules tend to align with their long axes (orientations $+\vec{n}$ and $-\vec{n}$ are equivalent). NLCs behave optically as a uniaxial birefringent material with $\vec{n}$ corresponding to the optical axis. If one travels along a path corresponding to a closed loop around a disclination, the orientation of $\vec{n}$ rotates by an angle $\boldsymbol{\Omega}$ :

$$
\Omega=2 \pi s,
$$

Max Planck Institute for Dynamics and Self-Organization (MPIDS), Am Fassberg 17, 37077 Göttingen, Germany. E-mail: christian.bahr@ds.mpg.de

$\dagger$ Electronic supplementary information (ESI) available: (I) Movie showing the formation of a zigzag disclination in a microfluidic channel. (II) Implementation of the Jones matrix method. See DOI: 10.1039/c7sm02286g

\# Present address: Physique et Matériaux, Université du Luxembourg, 162 A, avenue de la Faïencerie, L-1511, Luxembourg. where the strength $s$ of the disclination can take values $s= \pm \frac{1}{2}, \pm 1, \pm \frac{3}{2}, \ldots$. Fig. 1 gives the structure of the director field $\vec{n}(\vec{r})$ near a disclination for the two cases which are most frequently observed in real NLC samples $\left(s= \pm \frac{1}{2}\right)$. Disclination lines in NLCs have been recently employed as versatile tools for various applications, e.g., the architecture of micro-wires, ${ }^{10,11}$ the controlled positioning of colloidal objects, ${ }^{12-14}$ the use as templates for molecular self-assembly, ${ }^{15,16}$ or the use as soft rails for the transport of colloidal particles or droplets in microfluidic channels. ${ }^{17}$ Also fundamental aspects of disclination lines are still subject of current research despite the several decades which have passed since the seminal paper by Frank. ${ }^{7}$ For instance, the disclination loops entangling colloidal structures in nematic matrices ${ }^{18}$ are to be described rather as twisted ribbons than as simple lines. ${ }^{19,20}$

Disclination lines in NLCs are expected to occur in a large variety of structures. The two structures shown in Fig. 1 are
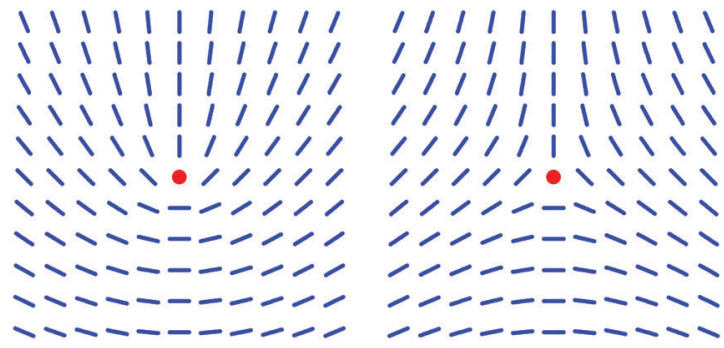

Fig. 1 Director fields near wedge disclinations of strength $s=+\frac{1}{2}$ (left) and $s=-\frac{1}{2}$ (right). The disclination line, marked by the red dot, is perpendicular to the plane of the figure. 


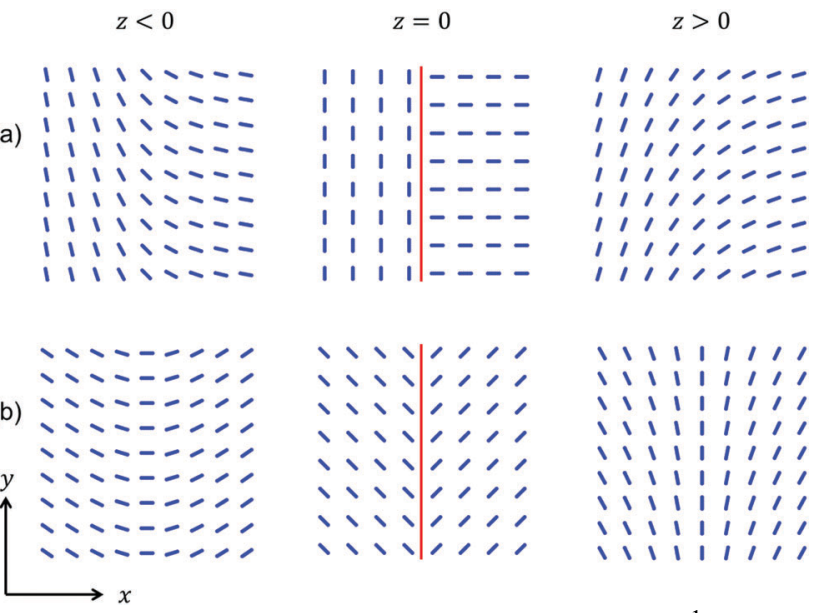

Fig. 2 Two examples of twist disclinations of strength $s=\frac{1}{2}$. The local orientation of the director is described by eqn (4), the upper row (a) shows the case $\varphi_{0}=0$, the lower row (b) is obtained with $\varphi_{0}=\frac{\pi}{4}$. The disclination line (red) is in the $x y$ plane and along $y, \vec{n}$ rotates around $z$. Besides the plane containing the disclination (middle panels) a plane above and a plane below the disclination is shown. Note that configuration (b) possesses a certain symmetry with respect to the $y z$ plane which is not present for configuration (a).

examples of wedge disclinations, i.e., the axis around which $\vec{n}$ rotates is parallel to the disclination line and $\vec{n}$ is confined to planes perpendicular to the line. Another type of disclination are twist disclinations. In that case, $\vec{n}$ rotates around an axis perpendicular to the disclination line and is confined to planes parallel to the line (Fig. 2). Mixed structures between pure wedge and twist also exist, and one can continuously transform a wedge disclination into a twist disclination of the same strength. ${ }^{1}$ The different structures are topologically equivalent and possess the same energy if elastic isotropy is assumed (i.e., if the elastic constants $K_{1}, K_{2}$ and $K_{3}$, describing splay, twist and bend deformations respectively, are assumed to be of equal size). In common NLC compounds, however, the twist elastic constant $K_{2}$ is significantly smaller than $K_{1}$ and $K_{3}$. Thus, by continuum theory arguments twist disclinations are expected to be preferred compared to wedge disclinations. ${ }^{21,22}$ Experimentally, however, it seems that wedge disclinations are preferred. Twist disclinations have been observed so far only in samples containing oppositely twisted domains generated by special boundary conditions, ${ }^{23}$ in NLC droplets sandwiched between two plates, where the disclination forms a loop near the meniscus to the air interface, ${ }^{24}$ or in samples in which electric field effects have generated a twisted domain in an planar environment. ${ }^{25}$ To the best of our knowledge, a continuous transformation of a wedge to a twist disclination (or vice versa) has been observed only in numerical simulations. ${ }^{26,27}$

In the present paper, we study disclination lines in microfluidic channels. The configuration of the director field $\vec{n}(\vec{r})$ of a NLC in microfluidic channels ${ }^{28}$ is determined by the anchoring of $\vec{n}$ on the channel walls (i.e., the preferred orientation of $\vec{n}$ in the NLC layer in contact with the wall), the flow of the NLC, and possible external fields influencing the orientation of $\vec{n}$.
We have recently shown that the interplay between anchoring, flow and an applied electric field enables an enhanced control of disclinations in the channel, resulting, e.g., in a possibility to interrupt and reconnect a disclination line. ${ }^{29}$ During the experiments described in ref. 29 we noticed that the disclination line adopts a zigzag shape at high electric field strength. We therefore conducted a detailed study of the structural transformations of disclination lines in microfluidic channels under the influence of flow and electric field and report here our results. The anchoring conditions on the channel walls are designed in a way which enforces, in the absence of flow, the formation of a wedge disclination along the channel axis. A flow of the NLC through the channel is found to transform the wedge disclination to the twist configuration. If in addition an electric field is applied, a second transformation of the disclination to a zigzag shape is observed. The threshold field strength for inducing the zigzag instability depends on the flow velocity of the NLC. The experimental observations are interpreted by comparison with energy calculations and simulations of microscopy images (calculated with the Jones matrix method) based on simple model director fields.

\section{Materials and methods}

Microfluidic devices are prepared by standard soft lithography methods. ${ }^{30}$ We use simple linear channels with dimensions between $100 \mu \mathrm{m}$ and $300 \mu \mathrm{m}$ in width and $20 \mu \mathrm{m}$ to $50 \mu \mathrm{m}$ in depth. The channels are generated as a relief in the surface of a poly(dimethysiloxane) (PDMS) block which is placed on a flat glass substrate, i.e., the channel possesses three PDMS walls and one glass wall (Fig. 3, top). On each side of the main channel an auxiliary channel runs parallel to the main channel. The two auxiliary channels are filled with a conductive material and enable the application of an electric field perpendicular to the axis of the main channel. We apply sinusoidal AC electric fields with a frequency of $1 \mathrm{kHz}$ and a field strength amplitude

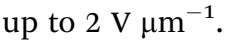

We use the common liquid crystal compound 4-pentyl-4'cyanobiphenyl $(5 \mathrm{CB})$ which is in the nematic state at room temperature. The flow of $5 \mathrm{CB}$ through the microchannel is generated by a gas-tight microliter syringe which is driven by a gear pump (neMESYS, Cetoni), allowing a control of the volumetric flow rate with a precision of $1 \mathrm{nl} \mathrm{h}^{-1}$. The volumetric flow rate is tuned in order to obtain a flow velocity with a value between $50 \mu \mathrm{m} \mathrm{s}^{-1}$ and $600 \mu \mathrm{m} \mathrm{s}^{-1}$ in the channel center.

The anchoring conditions for the nematic director $\vec{n}$ on the channel walls are as follows. On the surface of the three PDMS walls the anchoring is homeotropic (i.e., $\vec{n}$ is perpendicular to the wall), which is the intrinsic anchoring condition of a PDMS surface at ambient conditions. ${ }^{31}$ On the surface of the glass wall uniform planar anchoring (i.e., $\vec{n}$ is parallel to the wall and along a certain direction) is implemented by treating the glass surface with poly(tetrafluoroethylene) (PTFE): a block of PTFE is slowly moved at elevated temperature $\left(\approx 200{ }^{\circ} \mathrm{C}\right)$ on the glass surface, leaving behind a thin PTFE layer inducing uniform planar anchoring along the moving direction. ${ }^{32}$ The direction 


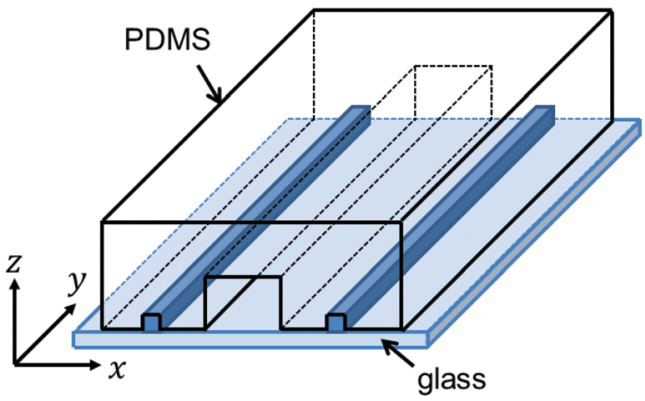

PDMS

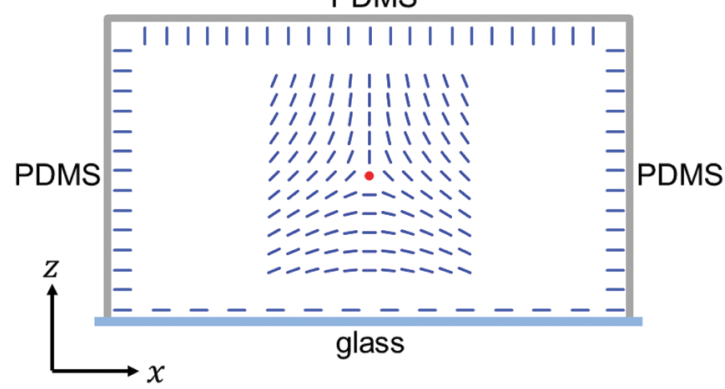

Fig. 3 Top: Schematic of a PDMS/glass microfluidic channel. A PDMS block, containing the channel pattern as a relief in its surface, is placed on a glass substrate. Two auxiliary channels, filled with low-melting solder, run parallel to the main channel and enable the application of an electric field perpendicular to the main channel. (Note that, in contrast to the above drawing, the auxiliary channels possess the same height as the main channel, resulting in a nearly homogeneous field across the channel.) Bottom: Anchoring conditions of the nematic director $\vec{n}$ on the channel walls ( $\vec{n}$ is parallel to the glass wall and along $x$, and perpendicular to each PDMS wall), enforcing the formation of a wedge disclination along the channel axis. For illustration, the cross section of the director field around a wedge disclination of strength $-\frac{1}{2}$ is shown (see right panel of Fig. 1), a wedge disclination of strength $+\frac{1}{2}$ would also meet the anchoring conditions.

of the uniform planar anchoring is chosen to be along $x$ (see Fig. 3, bottom), which is perpendicular to the channel axis.

Polarizing optical microscopy is done employing a Nikon Eclipse LV100 POL microscope, for fluorescence confocal polarizing microscopy a Leica TCS-SP2 microscope is used and the 5CB is doped with a small amount (0.01 wt\%) of the dye Nile red. All experiments and measurements are conducted at ambient temperature $\left(22{ }^{\circ} \mathrm{C}\right)$. Throughout the article, the coordinate system defined in Fig. 3 (top) is used: $y$ is the direction of the channel axis and the flow, $x$ is the direction from one side wall to the other and of the applied field and $z$ is the direction from the bottom (glass) wall to the top wall.

\section{Experimental observations}

We first give an overview of the polarizing microscopy observations, starting with the filled channel under the condition of zero flow and zero electric field. The channel is filled with $5 \mathrm{CB}$ in the nematic state at a low flow rate, corresponding to $\approx 50 \mu \mathrm{m} \mathrm{s}^{-1}$. During the filling of the channel a disclination line forms immediately which runs from the meniscus at the progressing $5 \mathrm{CB}$ /air interface approximately along the central axis of the channel. After completing the filling process the flow is stopped and the channel is let to equilibrate for at least $1 \mathrm{~h}$. During this period, the disclination approaches either of the side walls, approximately with equal probability, leading finally to a configuration in which the disclination runs very close to one of the side walls with eventually crossing the channel from one side wall to the other. Fig. 4(a) shows a micrograph of such a crossing of the disclination line between the side walls for the condition of zero flow and zero field. The micrograph is recorded with crossed polarizers and a $\lambda$ plate. Because the anchoring conditions confine $\vec{n}$ to the $x z$ plane and the polarizers are aligned along $x$ and $y$, the NLC in the channel appears optically isotropic, giving rise to the magenta color in the micrograph. Only near the crossing disclination line $\vec{n}$ deviates from the $x z$ plane, causing the bright appearance around the crossing line: as was shown in ref. 33, a wedge disclination which makes a non-perpendicular angle to the far field direction of $\vec{n}$ leads to a local deviation of $\vec{n}$ from the far field orientation (i.e., in our case to a deflection out of the $x z$ plane). The preference of a close distance between disclination and channel wall for the condition of zero flow has been already described in ref. 17 . We show in Section 4 that the reason for this behaviour is a smaller elastic energy compared to a position of the disclination in the channel center.

Fig. 4(b) shows a micrograph of the channel in the presence of a medium flow rate, corresponding to a flow velocity
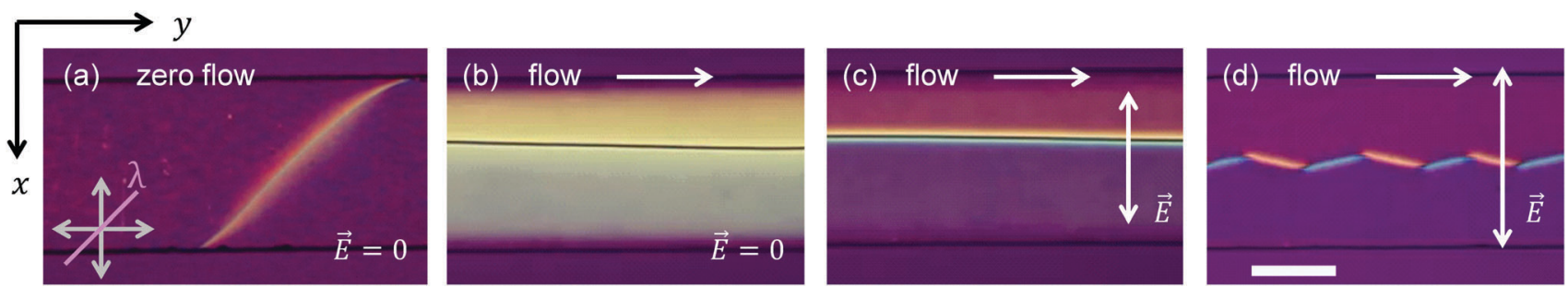

Fig. 4 Micrographs (crossed polarizers along $x$ and $y$ and diagonal $\lambda$ plate) showing the top view on a channel (200 $\mu \mathrm{m}$ wide, $20 \mu \mathrm{m}$ deep) under various conditions. (a) Zero flow and zero field, the disclination line is located very close to one of the side walls and is visible only when it crosses from one wall to the other. (b) Medium flow rate (flow velocity $\approx 200 \mu \mathrm{m} \mathrm{s}^{-1}$ in the channel center) and zero field, the disclination line is now located in the center and adopted the configuration of a twist disclination (see text). (c) Same flow rate as in (b) and electric field $E=0.5 \mathrm{~V} \mu \mathrm{m}^{-1}$ applied, in the major part of the channel $\vec{n}$ is approximately parallel to $\vec{E}$, deformations resulting from the disclination line are restricted to narrow regions next to the line. (d) Same flow rate as in (b) and electric field $E=1.5 \mathrm{~V} \mu \mathrm{m}^{-1}$ applied, the disclination has adopted a zigzag shape (see text). Scale bar corresponds to $100 \mu \mathrm{m}$. 
of $\approx 200 \mu \mathrm{m} \mathrm{s}^{-1}$ in the channel center. In a shear flow, the equilibrium orientation of $\vec{n}$ is approximately along the flow direction (more details will be given in Section 4), i.e., the flow in the microchannel (along $y$ ) is competing with the anchoring conditions (which favor $\vec{n}$ being in the $x z$ plane). We find that in the presence of a flow the disclination line is now visible in the central region of the channel. The magenta colour is replaced, except near the side walls, by a bright appearance of the NLC between crossed polarizers indicating that $\vec{n}$ is no longer confined to the $x z$ plane. If we observe the channel between parallel polarizers (and without $\lambda$ plate) we find extinction (except near the side walls), i.e., the transmitted light is linearly polarized with the plane of polarization being perpendicular to the analyzer. This observation indicates the presence of a twist deformation in the director field, rotating the plane of polarization of the incident light by $\approx \pi / 2$ during the passage through the channel. Rotations of the analyzer indicate in addition that the sense of rotation of the polarization plane is opposite on each side of the central disclination line and that the amount of rotation decreases towards the side walls (Fig. 5). All these optical observations are not compatible with the director field around a wedge disclination running along the channel axis. Rather, the optical properties suggest the presence of a twist disclination. An obvious qualitative argument why the presence of a flow induces a transformation to a twist disclination is that for a twist disclination at least some of the local directors possess an orientation approximately along the flow direction (y) whereas for a wedge disclination all local directors are perpendicular to the flow. We will explore this argument further in Section 4. We will also show that of the two configurations of twist disclinations shown in Fig. 2, in the presence of a flow the configuration is favoured which possesses mirror symmetry with respect to the $y z$ plane (shown in Fig. 2(b)). One should also have in mind, that the schemes of the director field shown in Fig. 2 (and also in Fig. 3, bottom) refer only to the volume part of the channel while close to the walls transition layers exist in which the director field is additionally deformed in order to meet the anchoring conditions on the walls.

We return to the discussion of the micrographs shown in Fig. 4. The NLC 5CB possesses a positive dielectric anisotropy, i.e., the preferred orientation of $\vec{n}$ would be parallel to an applied electric field $\vec{E}$. In our experiment, we apply the field along $x$, perpendicular to the flow direction, and we thus expect that $\vec{n}$ reorients towards the $x$ direction for sufficiently high field strength. When we switch on the field and increase the field strength (while keeping the flow rate constant and at the same level as in Fig. 4(b)), the bright-appearing areas, which extend at zero field from the central disclination line almost up to the side walls, contract themselves to narrow stripes which possess different colours and are located directly at both sides of the disclination line (Fig. 4(c)). The major part of the channel adopts almost the initial magenta colour (Fig. 4(a)) corresponding to the apparent optical isotropy which results if the optical axis is within the plane of polarization of the incident light. Subtle differences in the hue between both sides of the channel, even at larger distances from the disclination, indicate that the local
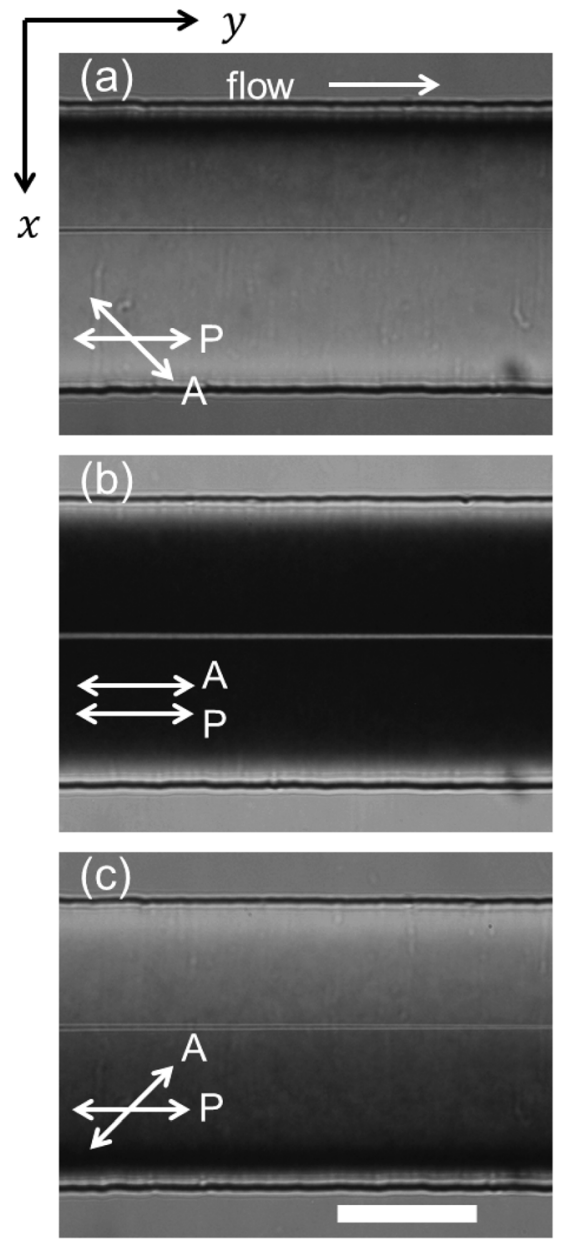

Fig. 5 Micrographs (polarizers not crossed, without $\lambda$ plate) showing the top view on the channel, same conditions as for Fig. 4(b). For polarizer and analyzer oriented parallel (panel (b)), extinction is observed for the major part of the channel, indicating the presence of $\pi / 2$ twisted structures in the director field. The change of the pattern on rotating the analyzer in different directions (panel (a) and (c)) indicates that the twist decreases towards the side walls and possesses opposite sense on each side of the disclination line. Scale bar corresponds to $100 \mu \mathrm{m}$.

directors are not completely within the $x z$ plane. It is obvious, however, that the director field deformation which results from the twist disclination line is restricted to a region with a size of a few $\mu \mathrm{m}$ around the disclination.

A second effect of the application of an electric field concerns the vertical position of the disclination line in the channel. Fig. 6 shows the distance $d$ of the disclination line from the planar orienting channel wall (i.e., the glass wall at the bottom) as a function electric field strength for a $20 \mu \mathrm{m}$ deep channel. The data are determined under the condition of a constant flow rate (velocity $\approx 200 \mu \mathrm{m} \mathrm{s}^{-1}$ in the channel center) by fluorescence confocal polarizing microscopy. While at zero field the disclination is located closer to the glass wall with $d \approx 7 \mu \mathrm{m}$, the position shifts towards the top wall with increasing field strength. At moderate field strength $\left(E \approx 0.15 \mathrm{~V} \mu \mathrm{m}^{-1}\right)$ the disclination is about $4 \mu \mathrm{m}$ away from the top wall $(d \approx 16 \mu \mathrm{m})$. The vertical shift of the disclination line is easily to understand qualitatively (Fig. 7): in the 


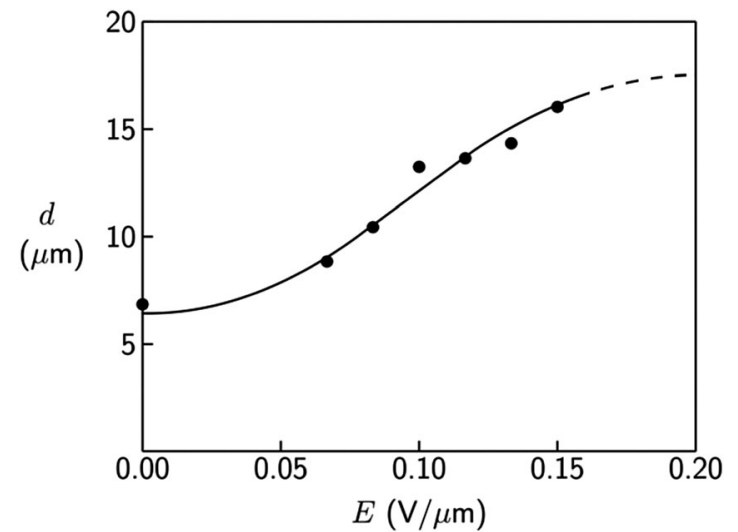

Fig. 6 Distance $d$ between the disclination line and the bottom (glass) wall of the channel as function of a applied electric field strength $E$ under the condition of constant flow rate (flow velocity $\approx 200 \mu \mathrm{m} \mathrm{s}^{-1}$ in the channel center). The line is just a guide to the eye. Larger distances than $\approx 16 \mu \mathrm{m}$ cannot be reliably determined with our confocal microscopy setup.

presence of flow and at zero field, a position near the bottom wall (glas wall) is preferred because then in a major part of the channel $\vec{n}$ can align approximately along the flow direction. If an electric field is applied perpendicular to the flow and its strength is high enough to overcome the aligning effect of the flow, the disclination line shifts towards the top wall because then in a major part of the channel $\vec{n}$ can align approximately along the field direction. We will explore this argument further in Section 4.

When we continue to increase the strength of the applied field the straight disclination line adopts a zigzag shape (Fig. 4(d), see also the movie provided as ESI $\dagger$ ). For the flow rate used in the experiment shown in Fig. 4, the disclination line in its straight configuration is stable for field strengths up to $\approx 1 \mathrm{~V} \mathrm{\mu m}^{-1}$ and tranforms to the zigzag configuration at higher field strengths. As shown in Fig. 8, the magnitude of the threshold field appears to increase linearly with the flow rate.

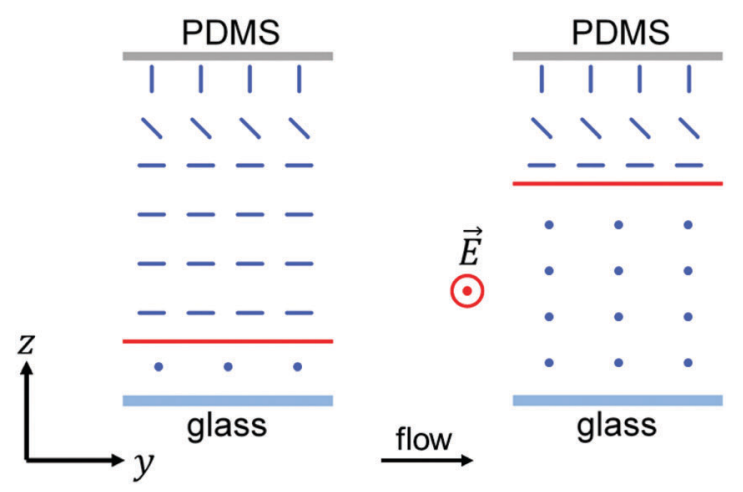

Fig. 7 Schemes of local director orientation (blue rods, dots indicate an orientation perpendicular to the plane of the figure). Shown are side views of the channel, i.e., cross sections parallel to the $y z$ plane containing the disclination line (red line). For both schemes a flow along $y$ is assumed. Without electric field (left panel), a position of the line near the bottom wall is favourable, application of an electric field (right panel) shifts the line towards the top wall.
After the transformation to the zigzag configuration, the complete zigzag pattern travels in the downstream direction with a velocity $v \approx \frac{1}{4} v_{\text {center }}\left(v_{\text {center }}\right.$ denotes the flow velocity of the NLC in the channel center). We should note that the zigzag configuration of the disclination can be induced by the field also at zero flow (see grey data points at $v=0$ in Fig. 8): when at zero flow the applied field is increased above $\approx 0.4 \mathrm{~V} \mu \mathrm{m}^{-1}$, the disclination line is detaching from the side wall of the channel and moves slowly towards the center. The line appears to be in the zigzag configuration already when it is detaching from the side wall. However, because the line is hardly to observe in the microscope before it detaches from the side wall (see Fig. 4(a)), we concentrate here on the case where the zigzag shape develops on a disclination which is stabilized by the presence of a flow in the channel center where it is well observable.

Zigzag instabilities of disclination lines have been described already in the 1980s, in biaxial lyotropic NLCs ${ }^{34,35}$ and also in uniaxial thermotropic $\mathrm{NLCs}^{35,36}$ (to which the compound 5CB of the present study belongs) in flat capillaries or Hele-Shawlike cells. More recently, zigzag shaped disclinations have been observed also in NLCs confined to microwrinkle grooves ${ }^{12,37}$ and were theoretically studied within a Landau-de Gennes model. ${ }^{38}$ In these studies ${ }^{12,34-38}$ it was argued that the elastic anisotropy of NLCs is at the origin of the zigzag disclinations: because twist deformations of the director field cost significantly less elastic energy than splay or bend deformations, disclination lines form under the boundary conditions of the above mentioned studies in the twist configuration and release some twist energy by inclining and forming the zigzag pattern.

Zigzag instabilities were also observed for $\chi$ disclination lines in cholesteric liquid crystals. The cholesteric phase is the chiral version of the nematic phase: it exhibits a spontaneous twist along one spatial direction, resulting in a helical superstructure in the director field. A $\chi$ disclination line separates two domains which differ by the amount of $\pi$ in their total twist. When a cholesteric phase was brought close to the transition to a smectic A phase, under certain conditions a zigzag instability of a

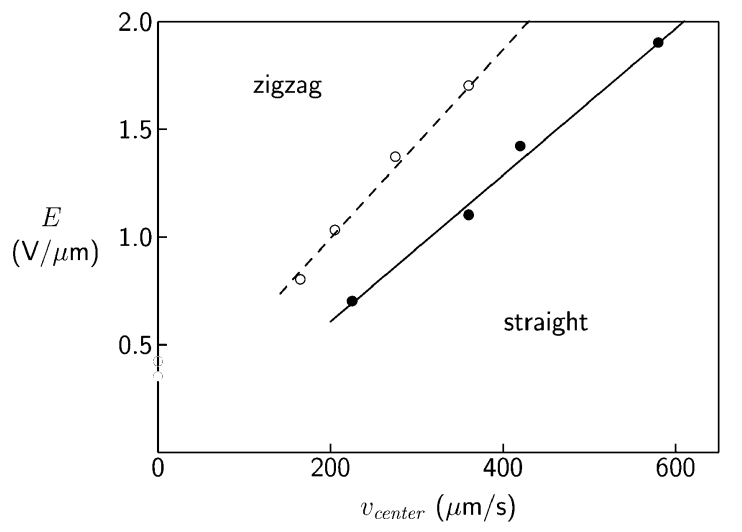

Fig. 8 Threshold field strength $E$ for the induction of the zigzag configuration as function of the flow rate ( $v_{\text {center }}$ : flow velocity in the channel center) for two different channel dimensions ( $\circ$ : width $200 \mu \mathrm{m}$, depth $25 \mu \mathrm{m}$, •: width $200 \mu \mathrm{m}$, depth $50 \mu \mathrm{m})$. 
$\chi$ disclination was observed ${ }^{39}$ which was explained through the divergence of the bend elastic constant at the transition. Two $\chi$ disclinations can join to form a double line (separating two twist domains differing by $2 \pi$ ) which was observed to show a zigzag instability in the presence of a magnetic field. ${ }^{40}$

Let us return to our study of disclinations in the conventional (nonchiral) nematic phase. Our experiment offers the unique possibility to observe the disclination line before and after the formation of the zigzag structure, i.e., to directly observe the structural transformation itself. The most prominent features of the transformation are the change of the colour pattern around the disclination line and the change of the position of the line relative to the colour pattern: for the straight configuration two narrow stripes, one on each side of line, are observed which exhibit different colours, suggesting the existence of twisted domains with opposite sense on each side of the disclination. In the zigzag configuration a coloured stripe exists only on one side of the disclination, with the side and the colour alternating between the zigs and the zags. The initial step of the transformation appears to be that one of the two coloured stripes slightly grows in width, thereby pushing the line across the second coloured stripe which decreases in width and disappears. Almost simultaneously, the line starts to incline and the zigzag pattern is formed (see movie provided as ESI $\dagger$ ). We will discuss possible structures in the following section.

\section{Comparison with model director fields}

The experimental observations presented in the previous section suggest the following scenario: at zero flow and zero field a wedge disclination is present near one of the side walls of the channel. A flow induces a transformation to a twist disclination which is located near the channel center. The application of an electric field quenches the twisted regions of the director field into narrow stripes close to the disclination. At lower field strengths, a straight symmetric configuration exists with two twist domains of opposite sense on both sides of the disclination. At higher field strengths, a configuration is induced in which the director field is no longer symmetric with respect to the disclination and the zigzag structure is formed. In this section, we calculate the energy of simple model structures, based on wedge and twist disclinations, in order to support the above described scenario. Additional confirmation will be provided by comparing the experimental polarizing micrographs with numerically calculated images obtained by applying the Jones matrix method on the model structures.

For the following calculations we define a model sample with dimensions $100 \times 100 \times 100 \mu \mathrm{m}^{3}$ and use the coordinate system shown in Fig. 3 , i.e., the flow direction is along $y$ and the electric field direction along $x$. The origin of the coordinate system is placed in the channel center. The disclination line is located on the $y$ axis. We divide each spatial dimension into 512 sections, resulting in $512^{3}$ lattice points and corresponding voxels. The orientation of the local director is described by the polar angle $\theta$, denoting the angle between $\vec{n}$ and the $z$ axis and the azimuthal angle $\varphi$, denoting the angle between the projection of $\vec{n}$ onto the $x y$ plane and the $x$ axis:

$$
\vec{n}=\left(\begin{array}{c}
\sin \theta \cos \varphi \\
\sin \theta \sin \varphi \\
\cos \theta
\end{array}\right) .
$$

The director field around a wedge disclination along $y$ is described by

$$
\theta=s \arctan \left(\frac{z}{x}\right)+\theta_{0} \quad \text { and } \quad \varphi=0
$$

and a twist disclination along $y$ is obtained with

$$
\theta=\frac{\pi}{2} \quad \text { and } \quad \varphi=s \arctan \left(\frac{z}{x}\right)+\varphi_{0}
$$

where $s$ is the strength of the disclination and $\theta_{0}$ and $\varphi_{0}$ are constant angles with arbitrary values, e.g., the scheme of the wedge disclination in Fig. 3(bottom) is obtained with $s=-\frac{1}{2}$ and $\theta_{0}=\frac{3 \pi}{4}$. At the channel walls, we fix the orientation of $\vec{n}$ with $\theta=\frac{\pi}{2}$ and $\varphi=0$ at the bottom and the two side walls and $\theta=0$ at the top wall, corresponding to the anchoring conditions shown in Fig. 3(bottom). We assume the existence of a transition layer at the channel walls in which the orientation of the director changes linearly from the orientation dictated by the anchoring on the walls to the orientation resulting from the structure of the director field in the channel volume. In the experiment, the width of the transition layer would depend on parameters such as flow rate or electric field strength. However, in order to keep our model simple, we set the width to a fixed mean value $(5 \mu \mathrm{m})$ based on polarizing microscopy observations at medium flow rates.

We consider three contributions to the total energy of our system: the elastic deformation of the director field, the potential energy in the electric field and the potential energy in the flow field. The elastic energy density $f_{\text {elastic }}$ is given by the usual combination of splay, twist and bend terms:

$$
f_{\text {elastic }}=\frac{K_{1}}{2}(\nabla \cdot \vec{n})^{2}+\frac{K_{2}}{2}[\vec{n} \cdot(\nabla \times \vec{n})]^{2}+\frac{K_{3}}{2}[\vec{n} \times(\nabla \times \vec{n})]^{2}
$$

with $K_{1}, K_{2}$ and $K_{3}$ being the elastic constants for the splay, twist and bend deformations of the director field. The energy density in the electric field, $f_{\text {electric }}$, depends on the relative dielectric anisotropy $\Delta \varepsilon_{\mathrm{r}}$ and the orientation of $\vec{n}$ with respect to the field $\vec{E}$ :

$$
f_{\text {electric }}=-\frac{1}{2} \varepsilon_{0} \Delta \varepsilon_{\mathrm{r}}(\vec{n} \cdot \vec{E})^{2}
$$

with $\varepsilon_{0}$ denoting the vacuum permittivity. While the calculation of the elastic energy and the energy in an electric field for a given director field is straightforward, a simple expression for the potential energy in a flow field, $f_{\text {flow, }}$ is less obvious. For the calculation of flow effects, usually the time evolution of the nematic tensor order parameter in the presence of flow and molecular field is considered employing a lattice Boltzmann algorithm (see, e.g., ref. 45 and references therein). Here, we 
use a much simpler approach based solely on the orientation of the director $\vec{n}$ with respect to a given shear flow. Let us first assume that $\vec{n}$ is within the shear plane. Then, we assign the energy $f_{\text {flow }}=0$ to the state where $\vec{n}$ is in the equilibrium orientation which is given by $\phi_{\text {eq }}=\arctan \sqrt{\frac{\alpha_{3}}{\alpha_{2}}}$. Here, $\phi_{\text {eq }}$ is the angle between $\vec{n}$ and the flow direction and $\alpha_{2}$ and $\alpha_{3}$ are coefficients of the viscous stress tensor, known as Leslie coefficients. ${ }^{46}$ If we want to rotate $\vec{n}$ within the shear plane to another orientation $\phi^{\prime}$, we have to perform work against the viscous torque $\Gamma$ caused by the flow. The torque is given as $\Gamma=\left(\alpha_{2} \sin ^{2} \phi-\alpha_{3} \cos ^{2} \phi\right) \nabla v$ with $v$ denoting the local flow velocity and $\phi$ the angle between the actual orientation of $\vec{n}$ and the flow direction ${ }^{46}$ (note that $\Gamma$ is defined per unit volume). We take the amount of work, which is needed to rotate $\vec{n}$ from $\phi_{\text {eq }}$ to $\phi^{\prime}$ (or which is released if $\vec{n}$ rotates from $\phi^{\prime}$ to $\phi_{\text {eq }}$ ) as a measure of the energy difference between a given orientation of $\vec{n}$ and the flow-aligned orientation, i.e., we consider it as a kind of potential energy density $f_{\text {flow }}$ in the flow field:

$$
f_{\text {flow }}=\int_{\phi_{\text {eq }}}^{\phi^{\prime}} \Gamma \mathrm{d} \phi .
$$

If $\vec{n}$ is not within the shear plane, the projection of $\vec{n}$ onto the shear plane, $\vec{n}_{\mathrm{p}}$, is considered instead of $\vec{n}$ and $f_{\text {flow }}$ is reduced by the factor $\left|\vec{n}_{\mathrm{p}}\right|$. For simplicity, we approximate the flow field in our model sample to the Poiseuille profile of a flow through a cylindrical tube possessing the same cross sectional area as our model $\left(10^{4} \mu \mathrm{m}^{2}\right)$. Eqn (7) just represents a minimal approach (which neglects, e.g., the mutual coupling between director orientation, viscosity, and flow) but, as is indicated by the results described in the following sections, it appeared sufficient for comparing the effects flow, electric field and elastic deformation for a given director field.

To obtain the total energy of our sample system, $F=\int_{V}\left(f_{\text {elastic }}+f_{\text {electric }}+f_{\text {flow }}\right) \mathrm{d} V$, the energy densities $f_{i}$ are calculated for each voxel, multiplied by the voxel volume and summed up. The spatial derivatives of the director components $n_{i}$ in eqn (5) are linearly approximated as $\frac{\Delta n_{i}}{\Delta d_{j}}$ where $\Delta d_{j}$ is the distance to the neighbouring lattice point in the corresponding spatial direction. Table 1 gives the values of the material constants used for our calculations. We should note that we do not aim at an iterative determination of the detailed structure corresponding to the global energy minimum under the respective conditions, rather we want to compare simple predefined model structures, based on wedge and twist disclinations in different

Table 1 Material constants of $5 \mathrm{CB}$

\begin{tabular}{lll}
\hline Quantity & Value & Reference \\
\hline$K_{1}$ & $6.4 \times 10^{-12} \mathrm{~N}$ & 41 and 42 \\
$K_{2}$ & $3.2 \times 10^{-12} \mathrm{~N}$ & 41 and 42 \\
$K_{3}$ & $9.0 \times 10^{-12} \mathrm{~N}$ & 41 and 42 \\
$\Delta \varepsilon_{\mathrm{r}}$ & 11 & 43 \\
$\alpha_{2}$ & $-90 \times 10^{-3} \mathrm{~Pa} \mathrm{~s}$ & 43 and 44 \\
$\alpha_{3}$ & $-4 \times 10^{-3} \mathrm{~Pa} \mathrm{~s}$ & 43
\end{tabular}

configurations, in order to obtain a general qualitative scenario corresponding to our experimental observations.

\subsection{Configuration in the absence of flow and field}

In the absence of flow and electric field, the anchoring conditions favour the formation of a wedge disclination. We calculate the elastic energy of our model sample with a wedge disclination according to eqn (3) with $s=-\frac{1}{2}$ and $\theta_{0}=\frac{3 \pi}{4}$ (this configuration matches the anchoring conditions as shown in Fig. 3, bottom). When varying the position of the disclination line, we find the lowest elastic energy if the disclination is close to one of the side walls and close to the top wall, in accordance with the experimental observations. As shown in Fig. 9, the elastic energy of our model sample decreases by more than $30 \%$ if the disclination is located near the upper left or upper right corner of the channel.

\subsection{Configuration in the presence of flow and absence of field}

When we switch on the flow, our experiment shows that the disclination line now appears in the central region of the channel and the optical properties suggest the configuration of a twist disclination. Here, we calculate the energies of a wedge and a twist disclination in our model sample in the presence of a flow. For the wedge disclination, we assume the same configuration as described above, while we assume for the twist disclination the symmetric configuration shown in Fig. 2(b) with $\varphi_{0}=\frac{\pi}{4}$. Fig. 10 shows the ratio of the total energies of the two configurations, $\frac{F_{\text {wedge }}}{F_{\text {twist }}}$, as a function of the flow velocity in the channel center $v_{\text {center. }}$ At zero flow and for small flow velocities, the wedge disclination possesses the lower energy because it matches better the anchoring conditions on the walls compared to the twist configuration. For $v_{\text {center }}$ above $\approx 25 \mu \mathrm{m} \mathrm{s}^{-1}$, the twist disclination is favoured because in some regions the local director is oriented approximately along the equilibrium orientation in the flow field.

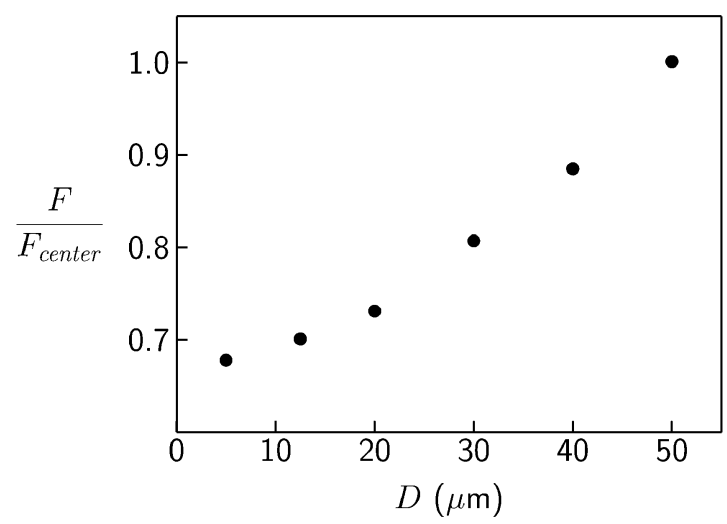

Fig. 9 Total energy $F$ as function of the position of a wedge disclination on the diagonal of the sample cross section under the condition of zero flow and zero electric field. $D$ denotes the distance to both a side wall and the top wall, i.e., $D=0$ corresponds to the upper left or upper right corner and $D=50 \mu \mathrm{m}$ to the center of the channel. The values of $F$ are scaled by $F_{\text {center }}=1.64 \times 10^{-14} \mathrm{~J}$ which is the value obtained for $D=50 \mu \mathrm{m}$. 


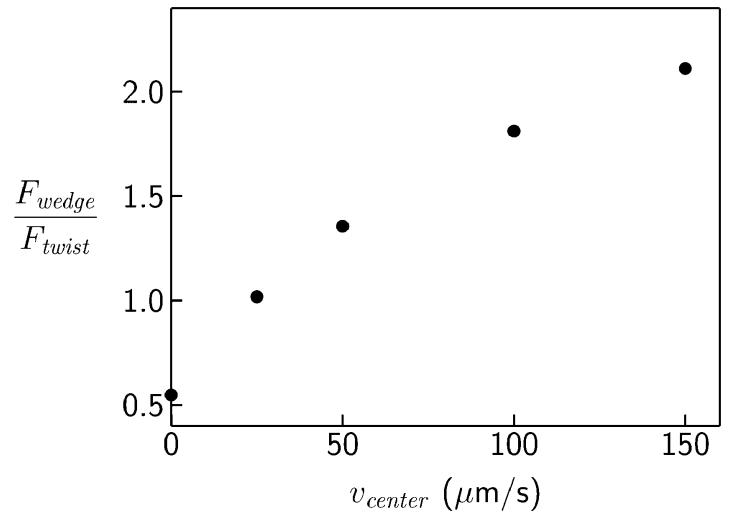

Fig. 10 Ratio of total energies of the model sample containing a wedge or a twist disclination, $\frac{F_{\text {wedge }}}{F_{\text {twist }}}$, as function of the flow velocity in the channel center $v_{\text {center }}$ (see text for details of the wedge and twist configurations).

For the twist disclination, we have also varied the offset angle $\varphi_{0}$ (see eqn (4)) in order to check if the symmetric configuration with $\varphi_{0}=\frac{\pi}{4}$ shown in Fig. 2(b) corresponds to an energy minimum. We find that this is indeed the case (see inset of Fig. 11). Furthermore, our experimental results indicate that, at finite flow and zero field, the twist disclination line is located in the lower half of the channel (see Fig. 6). We have varied in our model sample the position of the twist disclination along the $z$ axis and find an energy minimum for a $z$ position corresponding to $\approx \frac{1}{4}$ of the total channel height (Fig. 11). Thus, the numerical calculations confirm that the equilibrium configuration in the presence of a medium or stronger flow and at zero electric field consists of a twist disclination line in the symmetric

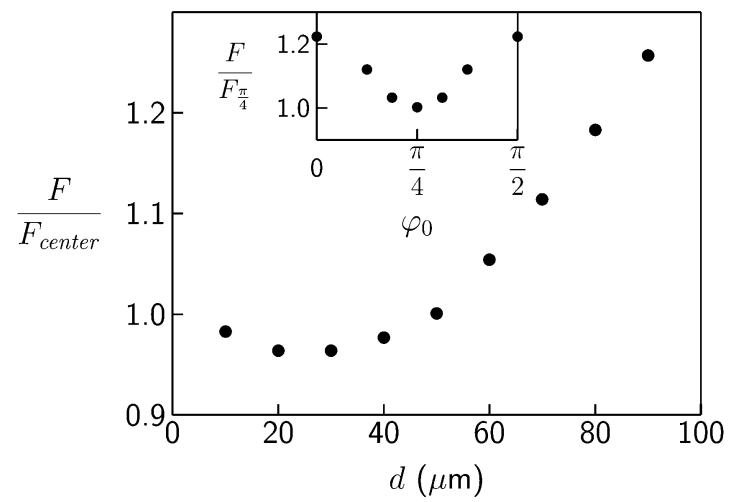

Fig. 11 Total energy $F$ of the model sample containing a twist disclination (with $\varphi_{0}=\frac{\pi}{4}$ ) as function of the distance $d$ between the disclination line and the bottom (glass) wall in the presence of a flow (flow velocity in the channel center was set to $v_{\text {center }}=100 \mu \mathrm{m} \mathrm{s}^{-1}$ ). The values of $F$ are scaled by Fcenter $=5.37 \times 10^{-14} \mathrm{~J}$ which is the value obtained for the center position with $d=50 \mu \mathrm{m}$. Inset: Total energy $F$ of the model sample containing a twist disclination (in the center position with $d=50 \mu \mathrm{m}$ ) as function of the offset angle $\varphi_{0}$. The values of $F$ are scaled by $F_{\frac{\pi}{4}}=5.37 \times 10^{-14} \mathrm{~J}$ which is the value obtained for $\varphi_{0}=\frac{\pi}{4}$. configuration $\left(\varphi_{0}=\frac{\pi}{4}\right)$ which is located approximately in the middle between the side walls and in the lower half of the channel.

\subsection{Configuration in the presence of flow and field}

When we switch on the electric field in the presence of a flow, polarizing microscopy indicates that the twisted domains on both sides of the disclination line shrink to narrow stripes located close to the line. Confocal microscopy shows in addition that the $z$ position of the line changes from the lower to the upper half of the channel. Both behaviours are readily reproduced for our model sample. We assume the presence of a twist disclination line in the symmetric configuration $\left(\varphi_{0}=\frac{\pi}{4}\right)$ running along $y$ and being located in the $y z$ plane. In order to simulate the lateral compression of the twisted domains near the disclination line, we introduce two uniform regions in which all local directors are oriented in the direction of the applied field. These regions extend over a distance $l_{\mathrm{u}}$ from the side walls towards the center of the channel where the disclination is located. We set the flow velocity to $v_{\text {center }}=100 \mu \mathrm{m} \mathrm{s}^{-1}$ and vary, for several values of the strength of the electric field, both $l_{\mathrm{u}}$ and the vertical position of the disclination line in order to find the configuration with the lowest energy. We find that the disclination line shifts already at moderate field strengths from the lower half of the channel to a position near the top wall (see inset of Fig. 12) while $l_{\mathrm{u}}$ shows a pronounced increase at somewhat higher field strengths (Fig. 12). At $E=1 \mathrm{~V} \mu \mathrm{m}^{-1}$, the lowest energy is obtained for $l_{\mathrm{u}} \approx 47 \mu \mathrm{m}$, i.e., the disclination-induced deformations of the director field are confined to two $3 \mu \mathrm{m}$ broad stripes near the disclination, and the disclination line is located in a close disctance $(\approx 2 \mu \mathrm{m})$ to the top wall. If we set $v_{\text {center }}$ to higher values, the behaviour remains qualitatively the same but shifts to larger field strengths. These findings are well in agreement with our experimental observations.

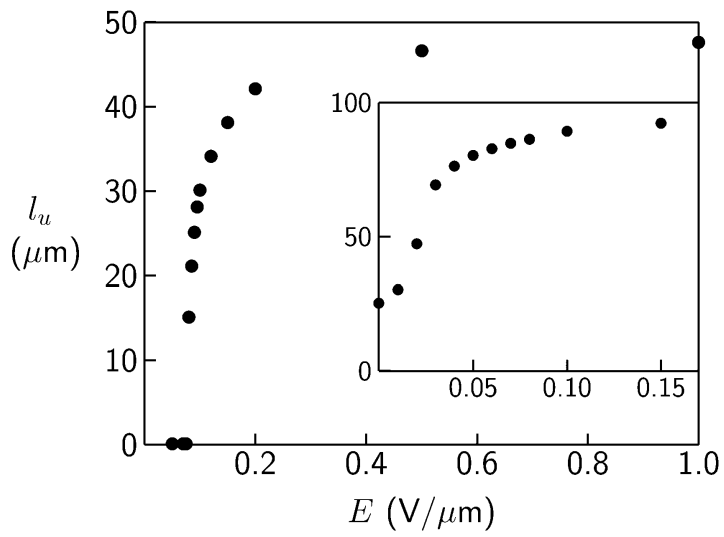

Fig. 12 Lateral width $l_{u}$ of the uniform field-aligned regions which grow with increasing electric field strength $E$ from the side walls towards the center of the channel and compress the twisted domains near the disclination. The flow velocity in the channel center was set to $v_{\text {center }}=100 \mu \mathrm{m} \mathrm{s}^{-1}$. Inset: Vertical position $d$ (the $y$ axis gives $d$ in $\mu \mathrm{m}$, at $d=0$ the bottom (glass) wall is located, at $d=100 \mu \mathrm{m}$ the top wall) of the disclination as function field strength $E$ (the $x$ axis gives $E$ in $\vee \mu m^{-1}$ ) for $v_{\text {center }}=100 \mu \mathrm{m} \mathrm{s}^{-1}$ 


\subsection{Origin of the zigzag structure}

In the following, we will argue that the zigzag structure of the disclination line is caused by an electric-field-induced change of the configuration of the twist disclination. The general director field of a twist disclination is described by eqn (2) and (4). Fig. 2 shows two examples of twist disclinations with strength $s=\frac{1}{2}$, differing in the value of the offset angle $\varphi_{0}$. Let us denote the configuration shown in Fig. $2 \mathrm{~b}\left(\varphi_{0}=\frac{\pi}{4}\right)$ as "symmetric" (because of its mirror symmetry with respect to the $y z$ plane) and the one shown in Fig. $2 \mathrm{a}\left(\varphi_{0}=0\right)$ as "asymmetric". Another asymmetric configuration is obtained for $\varphi_{0}=\frac{\pi}{2}$. If the director field is described solely by eqn (4) and not confined by any boundary conditions, the elastic energy and the potential energy in an electric field, applied in the $x y$ plane, are independent of the value of $\varphi_{0}$. This situation is significantly changed by the presence of the anchoring conditions imposed by the channel walls. If we calculate the potential energy in an electric field $(\vec{E} \| x)$ for our model sample, described in the second paragraph of Section 4, we find that the asymmetric configuration shows a sligthly lower energy than the symmetric configuration. On the other hand, we know already that in the presence of a flow the symmetric configuration is favoured (see inset of Fig. 11). Thus, by tuning electric field strength and flow velocity we expect the director field to switch between the symmetric (at large flow rate) and asymmetric (at large field strength) configuration. The zigzag structure appears because there are two asymmetric configurations, to which the symmetric configuration can transform with equal probability, and the asymmetric configurations can lower their elastic energy by making a finite angle (in the $x y$ plane) between the disclination line and channel axis.

Fig. 13 shows the elastic energy $F_{\mathrm{e}}$ of the symmetric $\left(\varphi_{0}=\frac{\pi}{4}\right)$ and the two asymmetric $\left(\varphi_{0}=0, \frac{\pi}{2}\right)$ model director

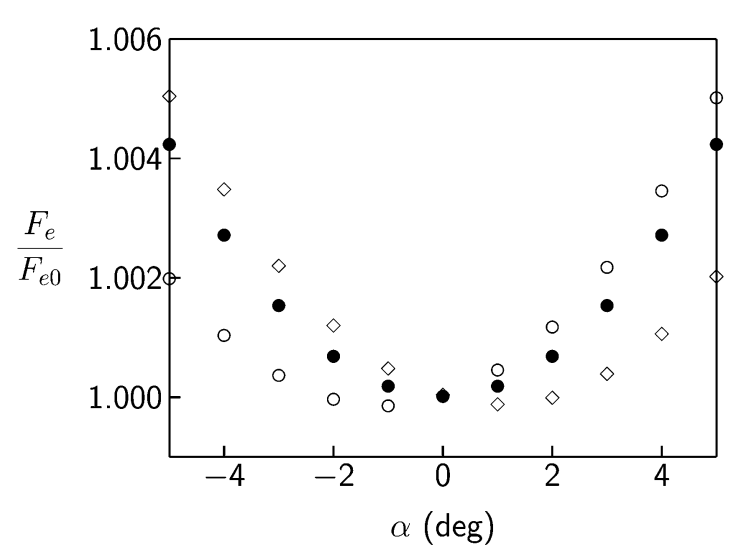

Fig. 13 Elastic energy $F_{\mathrm{e}}$ of the model sample as function of the angle $\alpha$ between disclination line and channel axis. $\bullet$ : symmetric configuration with $\varphi_{0}=\frac{\pi}{4}, \diamond$ : asymmetric configuration with $\varphi_{0}=0,0$ : asymmetric configuration with $\varphi_{0}=\frac{\pi}{2}$. The values of $F_{\mathrm{e}}$ are scaled by the values obtained for $\alpha=0\left(F_{\mathrm{e} 0}=3.01 \times 10^{-14} \mathrm{~J}\right.$ for the symmetric and $F_{\mathrm{e} 0}=$ $3.53 \times 10^{-14} \mathrm{~J}$ for the asymmetric configurations). fields, confined by the anchoring conditions on the channel walls, as a function of an angle $\alpha$ which may be seen as the angle between the channel axis $(y)$ and the disclination line in the $x y$ plane. In fact, for computing the data shown in Fig. 13 we did not change the position of the disclination. Rather, we rotated the in-plane direction of the planar anchoring on the bottom wall by the angle $\alpha$ from its initial direction (along $x$ ) and we changed the direction of the anchoring on the two side walls by the same angle $\alpha$, i.e., instead of rotating the disclination we rotated the boundary conditions (which is much easier to implement in our model). Whereas for the symmetric configuration the lowest elastic energy is obtained for $\alpha=0$, the two asymmetric configurations show their minimum for $\alpha \approx \pm 1^{\circ}$. Experimentally, we observe a value $\alpha \approx \pm 8^{\circ}$. Given the simplicity of our model, this deviation is not surprising.

We should note that we did not observe a clear relation between the experimental conditions and the parameters of the zigzag structure. Within the range, in which we have varied flow velocity, electric field strength, and channel dimensions, we always observed similar values for the angle between the zigs and zags and also for the periodicity of the zigzag structure along the channel axis. Only for the case of a change of the liquid crystal compound we observed a clear effect: we prepared a mixture of $5 \mathrm{CB}$ with its longer homolog $8 \mathrm{CB}$ (which is in the smectic state at room temperature). The composition of the mixture was chosen such that it exhibits a nematic to smectic-A transition slightly below room temperature, i.e., our experiment was done in the nematic state but close to ( $\approx 1 \mathrm{~K}$ or less) the transition to the smectic phase. Because the twist and bend elastic constants diverge at the nematic-smectic-A transition, the mixture can be expected to show larger elastic constants compared to pure 5CB. We found for the mixture significantly smaller values of both the zigzag angle and the zigzag periodicity. Obviously, the elastic constants of the liquid crystal play an essential role for the structure of the zigzag configuration.

We can give a rough estimation of the electric field strength which is necessary to transform, at a given flow rate, the symmetric configuration into the asymmetric. When we calculate the electric and flow parts, $F_{\text {elec }}$ and $F_{\text {flow }}$, of the total energy for our model sample we find for the difference between symmetric and asymmetric configurations the following relations:

$$
\begin{gathered}
\Delta F_{\text {elec }}=C_{\text {elec }} E^{2}, \\
\Delta F_{\text {flow }}=C_{\text {flow }} v_{\text {center }} .
\end{gathered}
$$

Here, $\Delta$ refers to the difference $F_{\text {sym }}-F_{\text {asym }}, E$ and $v_{\text {center }}$ denote electric field strength and flow velocity in the channel center and the constants are obtained as $C_{\text {elec }}=3.19 \times 10^{-25} \mathrm{~J} \mathrm{~m}^{2} \mathrm{~V}^{-2}$ and $C_{\text {flow }}=-6.16 \times 10^{-11} \mathrm{~J} \mathrm{~s} \mathrm{~m}^{-1}$ (these values are obtained for a fixed position of the disclination line in the channel center and for setting $l_{\mathrm{u}}=0$ regardless of the magnitude of $E$ or $v_{\text {center}}$ ). For $v_{\text {center }}=400 \mu \mathrm{m} \mathrm{s}^{-1}$, the influence of the electric field should outweigh that of the flow for $E \approx 0.3 \mathrm{~V} \mathrm{\mu m}^{-1}$ which is in the same order magnitude as the experimental values (see Fig. 8). From eqn (8) and (9) one expects the threshold field $E$ 
to vary as $\left(v_{\text {center }}\right)^{1 / 2}$ whereas our experimental data rather suggest a linear relation between $E$ and $v_{\text {center }}$ (see Fig. 8). However, we should note that eqn (8) and (9) just provide a first estimation because we did not take into account that, depending on the values of $E$ or $v_{\text {center }}$, the $z$ position of the disclination line changes and in large parts of the channel volume the director aligns parallel to $\vec{E}$.

The comparison between the experimental polarizing micrographs and calculated images, presented in the following section, provides another indication that the change between the symmetric and asymmetric configuration of the twist disclination is the origin of the zigzag structure.

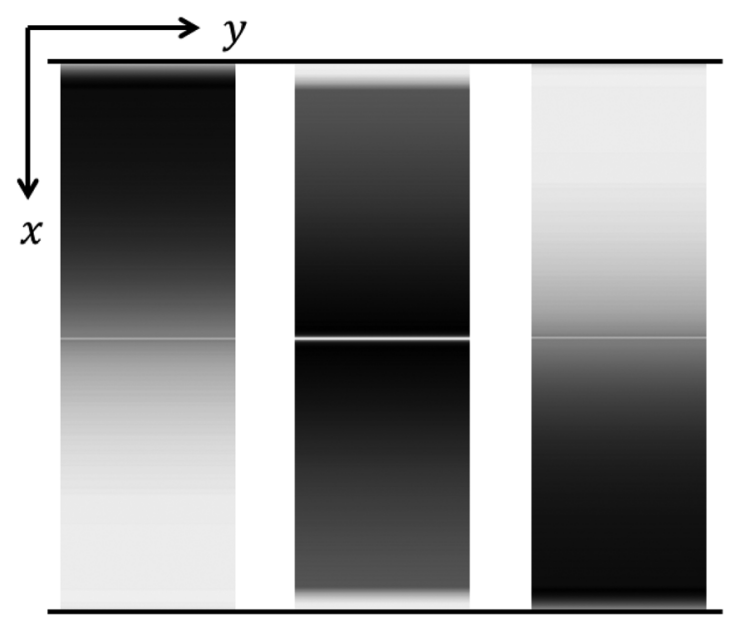

Fig. 14 Calculated images of polarizing micrographs (without $\lambda$ plate) for the model director field of a symmetric twist disclination as described in the text. The orientation of the polarizer is set along $y$ for all three images. Left image: Analyzer rotated by -40 degrees with respect to polarizer, middle image: analyzer parallel to polarizer, right image: analyzer rotated by +40 degrees with respect to polarizer. Horizontal black lines indicate the position of the channel side walls. Compare with the experimental micrographs shown in Fig. 5.

\subsection{Experimental and numerical polarizing micrographs}

The Jones matrix formalism ${ }^{47}$ allows the numerical calculation of polarizing micrographs provided the structure and optical properties of the sample are known. We apply this method to our model sample described in the second paragraph of Section 4. Technical details of our implementation of the Jones matrix method are given in the ESI. $\dagger$ Fig. 14 shows calculated images of polarizing micrographs of our model sample containing a twist disclination with strength $\frac{1}{2}$ in the symmetric configuration $\left(\varphi_{0}=\frac{\pi}{4}\right)$. The disclination line is along $y$ and located in the channel center, i.e., the director field is almost solely described by eqn (2) and (4) except for the transition layers near the channel walls where the anchoring conditions have to be met. For the calculation of the micrographs, the positions of polarizer and analyzer were set corresponding to the experimental conditions shown in Fig. 5. As expected for the case when polarizer and analyzer are set parallel (middle image in Fig. 14), extinction is observed close to the disclination where the director field describes an almost complete $\frac{\pi}{2}$ twist along $z$. The extinction becomes weaker with increasing lateral disctance to the disclination. Close to the side walls, a thin bright layer is obtained because of the homeotropic anchoring of the director. These results, and the images obtained for a rotation of the analyzer, agree well with experimental observations and confirm that the director field in our microchannel in the presence of flow and the absence of electric field corresponds essentially to a twist disclination with $\varphi_{0}=\frac{\pi}{4}$. Note that for a twist disclination in the asymmetric configuration $\left(\varphi_{0}=0\right.$ or $\left.\frac{\pi}{2}\right)$ significantly different images are obtained which do not match the experimental micrographs shown in Fig. 5.

Next we consider the change of the colour pattern at the electric-field-induced formation of the zigzag structure. On application of an electric field along $x$ the director aligns with the field in the major part of the channel volume and the
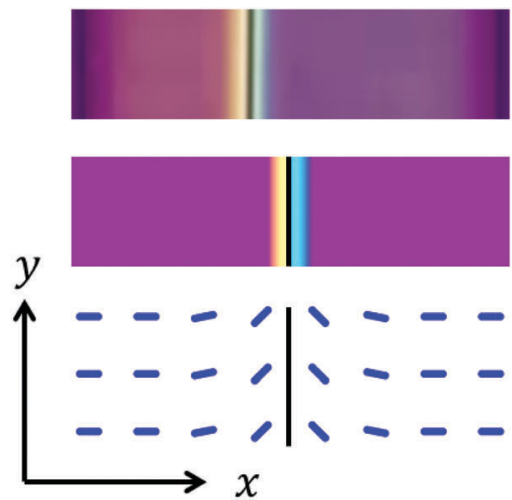
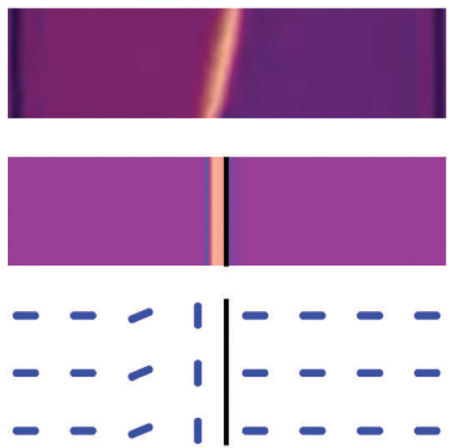
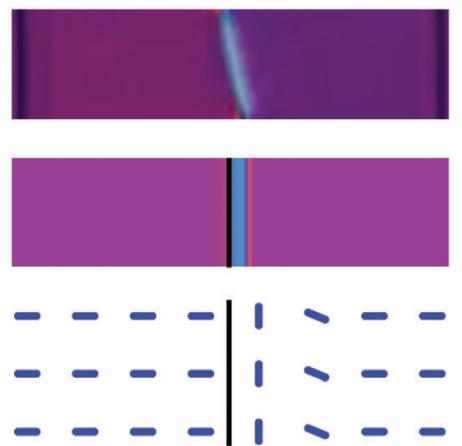

Fig. 15 Calculated images of polarizing micrographs (polarizer and analyzer along $x$ and $y$ and diagonal $\lambda$ plate) for the model director field near symmetric and asymmetric twist disclinations, confined to a narrow region around the disclination line, as described in the text. Top row: Experimental micrographs (same as in Fig. 4). Middle row: Calculated micrographs, the black line indicates the position of the disclination line. Bottom row: Schematics of the director field in the plane containing the disclination line (black line, note that the lateral scale of the schematics is not the same as in the micrographs but $\approx 10$ times expanded). Left panel: Symmetric configuration of the twist disclination $\left(\varphi_{0}=\frac{\pi}{2}\right)$. Middle and right panel: the two asymmetric configurations $\left(\varphi_{0}=0\right.$ and $\left.\frac{\pi}{2}\right)$. 
position of the disclination line shifts towards the top wall of the channel. As directly observed in the polarizing microscope (see Fig. 4c) and confirmed by the energy calculations presented above (see Fig. 12), the lateral extension of the region in which the director field is determined by the twist disclination shrinks to a size of few $\mu \mathrm{m}$. In order to reproduce the experimental micrograph shown in Fig. 4c with our model sample, we define a twist disclination in the symmetric configuration along $y$, located within the $y z$ plane at a distance $d_{\text {top }}$ from the top wall and assume that the director field is determined by the disclination within a region with an extension of $w-2 l_{\mathrm{u}}$ ( $w$ denoting the width $(100 \mu \mathrm{m})$ of our model sample) in $x$ direction and $2 d_{\text {top }}$ in $z$ direction (see also Fig. S3 in the ESI $\dagger$ ). In the remaining major part of the channel the director is aligned parallel to $x$ (direction of the applied field). We set $l_{\mathrm{u}}$ to $45 \mu \mathrm{m}$ and vary $d_{\text {top }}$ between $0.2 \mu \mathrm{m}$ and $2 \mu \mathrm{m}$. Regardless of the value of $d_{\text {top }}$, for the major area of the calculated images the magenta background, indicating (apparent) optical isotropy, is obtained, because in these regions the director (or its projection on the $x y$ plane) is aligned along $x$ (parallel to polarizer). On both sides of the disclination, two narrow coloured stripes are present. These colours depend strongly on the distance along $z$ over which the director varies and thus on the value of $d_{\text {top }}$ in our model sample. The best agreement between the experimental and the calculated micrograph is obtained for $d_{\text {top }}=0.6 \mu \mathrm{m}$ (see left panel of Fig. 15). If we change now the offset angle $\varphi_{0}$ (while all other conditions remain unchanged) in order to transform the director field into the two asymmetric configurations, we find that the experimental observations concerning the zigzag structure are well reproduced: a coloured stripe is now present mainly on one side of the disclination and the two colours of the two configurations resemble the experimentally observed colours (see middle and right panel of Fig. 15). These results confirm that the formation of the zigzag structure is caused by the preference of the asymmetric configurations of the twist disclination by the electric field.

\section{Conclusions}

We have presented a study of structural transformations of nematic disclination lines in microfluidic channels under the influence of anchoring, flow and electric field. The anchoring conditions on the channel walls are designed such that, in the absence of flow and field, the formation of a wedge disclination is enforced which is located close to one of the channel side walls. Our results show that a flow, which moves the disclination line to the channel center, transforms the wedge disclination to a twist disclination. It would be interesting to study this process in more detail because experimental studies of continuous transformations between wedge and twist disclinations have not been reported so far. Preliminary observations show that the time span in which the disclination becomes rebonded at the channel wall (and thus becomes unobservable) after the flow has stopped is of the order of $1 \mathrm{~min}$. Thus, by slowly decreasing the flow rate it might be possible to transform the flow-stabilized twist disclination continuously in a controlled way back to a wedge disclination and optical studies might elucidate how the director field changes its structure during the transformation.

A flow of sufficient strength stabilizes the twist disclination in the channel center. The director field around this flowstabilized twist disclination adopts a structure, characterized by the offset angle $\varphi_{0}=\frac{\pi}{4}$ (see eqn (4)), which is symmetric to the vertical channel mid-plane. The application of an electric field perpendicular to this plane has two independent effects. First, the vertical position of the disclination in the channel changes and the twisted regions of the director field become confined to narrow stripes near the disclination. Second, when the field strength reaches a certain threshold value, the symmetric configuration of the twist disclination becomes unstable with respect to two asymmetric configurations with offset angles $\varphi_{0}=0$ or $\frac{\pi}{2}$. The asymmetric configurations can decrease their elastic energy by adopting a small tilt angle to the channel axis, resulting in the formation of a zigzag structure. The great similarity of the appearance in a polarizing microscope suggests that the zigzag-shaped disclination observed in the present study and the zigzag disclinations observed recently in microwrinkle grooves ${ }^{12,37}$ possess similar structures. On the other hand, the formation process is clearly different: whereas in the previous studies ${ }^{12,34-37}$ the zigzag structure forms due to certain boundary conditions, the zigzag structure of the present study is the result of a complex interplay between anchoring, flow and electric field, which offers the unique possibility to actively switch between linear and zigzag configurations. Since it was already shown that colloidal particles become preferably trapped at the kinks of the zigzag disclinations, ${ }^{12}$ the active switching between linear and zigzag configurations might offer new possibilities for the controlled positioning and release of colloidal entities. From a more general viewpoint, we report a platform for the controlled generation of twist disclinations in different configurations. It would be interesting to study other NLCs which differ in their elastic and/or dielectric properties.

\section{Conflicts of interest}

There are no conflicts of interest to declare.

\section{Acknowledgements}

Stimulating discussions with Stephan Herminghaus and Takuya Ohzono are gratefully acknowledged.

\section{References}

1 M. Kléman and O. D. Lavrentovich, Soft Matter Physics: An Introduction, Springer, New York, 2003.

2 U. Tkalec, M. Ravnik, S. Čopar, S. Žumer and I. Muševič, Science, 2011, 333, 62-65.

3 S. Čopar, U. Tkalec, I. Muševič and S. Žumer, Proc. Natl. Acad. Sci. U. S. A., 2015, 112, 1675-1680. 
4 I. Muševič, M. Škarabot, U. Tkalec, M. Ravnik and S. Žumer, Science, 2006, 313, 954-958.

5 B. Senyuk, Q. Liu, S. He, R. D. Kamien, R. B. Kusner, T. C. Lubensky and I. I. Smalyukh, Nature, 2013, 493, 200-205.

6 I. Chuang, R. Durrer, N. Turok and B. Yurke, Science, 1991, 251, 1336-1342.

7 F. C. Frank, Discuss. Faraday Soc., 1958, 25, 19-28.

8 S. Chandrasekhar and G. S. Ranganath, Adv. Phys., 1986, 35, 507-596.

9 M. Kléman, Rep. Prog. Phys., 1989, 52, 555-654.

10 J.-B. Fleury, D. Pires and Y. Galerne, Phys. Rev. Lett., 2009, 103, 267801.

11 H. Agha, J.-B. Fleury and Y. Galerne, Eur. Phys. J. E: Soft Matter Biol. Phys., 2012, 35, 82.

12 T. Ohzono and J. Fukuda, Nat. Commun., 2012, 3, 701.

13 M. Cavallaro, M. A. Gharbi, D. A. Beller, S. Čopar, Z. Shi, R. D. Kamien, S. Yang, T. Baumgart and K. J. Stebe, Soft Matter, 2013, 9, 9099-9102.

14 H. Yoshida, K. Asakura, J. Fukuda and M. Ozaki, Nat. Commun., 2015, 6, 7180.

15 X. Wang, D. S. Miller, E. Bukusoglu, J. J. de Pablo and N. L. Abbott, Nat. Mater., 2016, 15, 106-112.

16 T. Ohzono, K. Katoh and J. Fukuda, Sci. Rep., 2016, 6, 36477.

17 A. Sengupta, C. Bahr and S. Herminghaus, Soft Matter, 2013, 9, 7251-7260.

18 M. Ravnik, M. Škarabot, S. Žumer, U. Tkalec, I. Poberaj, D. Babič, N. Osterman and I. Muševič, Phys. Rev. Lett., 2007, 99, 247801.

19 S. Čopar and S. Žumer, Phys. Rev. Lett., 2011, 106, 177801.

20 S. Čopar, T. Porenta and S. Žumer, Phys. Rev. E: Stat., Nonlinear, Soft Matter Phys., 2011, 84, 051702.

21 S. I. Anisimov and I. E. Dzyaloshinskii, Sov. Phys. JETP, 1973, 36, 774-779.

22 G. S. Ranganath, Mol. Cryst. Liq. Cryst., 1982, 87, 187-195.

23 C. J. Gerritsma, J. A. Geurst and A. M. J. Spruijt, Phys. Lett. A, 1973, 43, 356-358.

24 S. Thiberge, C. Chevallard, J. M. Gilli and A. Buka, Liq. Cryst., 1999, 26, 1225-1234.
25 K. S. Krishnamurthy, P. Tadapatri and W. Weissflog, Soft Matter, 2011, 7, 6273-6284.

26 J. Fukuda, Phys. Rev. E: Stat., Nonlinear, Soft Matter Phys., 2010, 81, 040701.

27 P. P. Avelino, F. Moraes, J. C. R. E. Oliveira and B. F. de Oliveira, Soft Matter, 2011, 7, 10961-10966.

28 A. Sengupta, S. Herminghaus and C. Bahr, Liq. Cryst. Rev., 2014, 2, 73-110.

29 H. Agha and C. Bahr, Soft Matter, 2016, 12, 4266-4273.

30 D. C. Duffy, J. C. McDonald, O. J. A. Schueller and G. M. Whitesides, Anal. Chem., 1998, 70, 4974-4984.

31 A. Sengupta, B. Schulz, E. Ouskova and C. Bahr, Microfluid. Nanofluid., 2012, 13, 941-955.

32 P. Hubert, H. Dreyfus, D. Guillon and Y. Galerne, J. Phys. II, 1995, 5, 1371-1383.

33 M. Buscaglia, G. Lombardo, L. Cavalli, R. Barberi and T. Bellini, Soft Matter, 2010, 6, 5434-5442.

34 Y. Galerne and L. Liébert, Phys. Rev. Lett., 1985, 55, 2449-2451. 35 M. Mihaïlovic and P. Oswald, J. Phys., 1988, 49, 1467-1475. 36 Y. Galerne, J. Itoua and L. Liébert, J. Phys., 1988, 49, 681-687. 37 T. Ohzono and J. Fukuda, Soft Matter, 2012, 8, 11552-11556. 38 N. M. Silvestre, J. M. Romero-Enrique and M. M. Telo da Gama, J. Phys.: Condens. Matter, 2017, 29, 014004.

39 A. Dequidt and P. Oswald, Eur. Phys. J. E: Soft Matter Biol. Phys., 2006, 19, 489-500.

40 Orsay Liquid Crystal Group, Phys. Lett. A, 1969, 28, 687-688. 41 J. D. Bunning, T. E. Faber and P. L. Sherrell, J. Phys., 1981, 42, 1175-1182.

42 G.-P. Chen, H. Takezoe and A. Fukuda, Liq. Cryst., 1989, 5, 341-347.

43 K. Skarp, S. T. Lagerwall and B. Stebler, Mol. Cryst. Liq. Cryst., 1980, 60, 215-236.

44 A. G. Chmielewski, Mol. Cryst. Liq. Cryst., 1986, 132, 339-352.

45 C. Denniston, D. Marenduzzo, E. Orlandini and J. M. Yeomans, Philos. Trans. R. Soc. London, Ser. A, 2004, 362, 1745-1754.

46 P. Oswald and P. Pieranski, Nematic and Cholesteric Liquid Crystals, Taylor \& Francis, Boca Raton, 2005, ch. B.III, pp. 173-242.

47 R. C. Jones, J. Opt. Soc. Am., 1941, 31, 488-493. 